\title{
A Model of Information Sharing Measurement in Integrated Supply Chain Networks
}

\author{
Asst. Prof. Dr. Fatih Çallı (Sakarya University, Turkey) \\ Asst. Prof. Dr. Sitkı Sönmezer (Beykent University, Turkey)
}

\begin{abstract}
In recent years, academic and industry areas are both show an interest in supply chain management (SCM). The globalization of competition, the reduce in product life cycle, time depending competition and quick exchanges make the firms think about re-designing their supply chains. With the view of these evolutions, firms decided that they must use information technologies in their SCM.

In future, information flow will be the key factor to determine the levels and the difference between firms. Information flow occurs with the integration of information technologies with the systems. The success factor for a firm is to add value from gathering, operating and dispatching information and develop the work model to have this value. Processing this model depends on achieving information sharing between supply chain members.

Information sharing is a critical factor between supply chain members. The data shared between supply chain members becomes useful information for the members.

So, determining the shared data and measuring this share is an important issue. This study includes information sharing measurement model between integrated supply chain members. The model starts with gathering data with a survey and continues converting these quantitative data's to qualitative data's by structured equations. The qualitative data's used in an artificial intelligence method, inductive learning. Rules extraction is made by an inductive learning method, RULESX. With these rules, information sharing among supply chain members can be measured and suggestions can be made to firms for improving their SCM's. This model is sector-independent and can be easily applied.
\end{abstract}

\section{Literature Overview}

The supply chain can be defined as a network connection between partners, such as suppliers and customers. Many researchers have seen that interdisciplinary cooperation in the supply chain is an important phenomenon. Kalakota and Robinson (2001) have demonstrated important developments in supply chain management, the mergers of their business and the successful exchange of information between the trade partners. Stevens (1989) argued that material flow management should be evaluated by three different perspectives in the development of integrated supply chain. He said all of the possibilities should work in the form of human power, financial movements, and system as a harmony. They point out these perspectives as strategic, tactical and operational perspectives.

Integration of Supply chain management ensures the following gains are achieved (Themistocleous et al 2004).

- Competition advantage

- $\quad$ Reduce transaction costs

- Ensuring coordination between better cooperation and supply chain partners

Many studies on strategies and techniques for development and design of supply chain management are included in the literature. In addition, several scientific studies include classification of supply chain management, analysis of the modeling and supply chain management. However, in recent years, the scientific studies on this subject have focused on information technology in the supply chain method. It is difficult to achieve a productive supply chain without knowledge technology. Because of the spread of suppliers to the entire world, an organization is important to combine internal and external activities. This requires a unified knowledge system for sharing information on the activities of adding value to the chain along the supply chain. Information technology is like a nervous system for supply chain management. There are many studies in the concept of supply chain related to information technology. Most of the literature discusses two views of one or two of the applications, such as strategies, tools and techniques. However, a comprehensive IT technology survey will be useful in determining the critical success factors of informational technologies for an integrated supply chain. Unfortunately, there is not enough interest from both researchers and practitioners in the application of IT technologies for design and efficient supply chain management (Guo et al., 2006).

- Integrated Supply Chain and Information Technologies

Global competition has resulted in rapid changes in time-varying competition and information technology transformation, and has caused companies to think about this subject again and change their strategies to restructure supply chains. Companies have made their own decisions with co-operation partners and co-ordination to provide supply chain deals. 
Stefansson (2002) conducted a study examining the benefits of advanced IT technologies for data sharing in small and medium-sized businesses in the supply chain. As a result of a total of 20 sample cases of transport and manufacturing companies, these firms have found that only those of small and medium-sized ones use only telephone and fax equipment as communication technologies, lacking basic technology systems. Referring to these types of companies the benefits of technologies such as EDI and the Internet, demonstrating that these data sharing efforts could be better co-ordinated.

Gunasekaran (2004) demonstrated the importance of information technology infrastructure in the supply chain and presented detailed literature research. The aim of this study is to determine the appropriate classification in the field of supply chain management and to develop a framework for information technology in supply chain management.

Simatupang and Sridharan (2001) stated that they first helped to acquire knowledge sharing, contract-based clarity. The contract is a contract of transfer payments, performance criteria, decision area areas, and the required level of sharing between the chain members in a particular market.

Secondly, the sharing of customer data allows the chain members to instantly intervene instinct in their marketplace. For example, the retailer may provide sales and customer information to the manufacturer. The manufacturer may use this information for the latest customer's behavior and demand growth.

Third, it facilitates the supply chain coordination of the supply chain of supply chain members. The goal is to improve the order process. Inventory information, order information, production scheduling information, and cost information are shared conversations (Simatupang and Sridharan 2001).

The profitability of a firm should be increased, or at least the profit expectancy should not be reduced due to the increased visibility. This is supported by many applications and studies in supply chain management. For example, performing CPFR and VMI applications depends on the supply chain elements to share information such as order and inventory.

Lee and Wang (1998) define a total of 5 shared information, including inventory, sales, demand estimation, order and production scheduling information. In their studies, they introduced this information and why it was shared on an academic platform by explaining industrial samples. From this, information transfer model has developed a total of 3 alternative system models as a third-party model and information hub model.

Gavirneni et al. (1999) addressed a supplier with a two-stage supply chain, and a retailer. In this model, the retailer is facing a number of favorable demands and these demands meet the supplier policy at any time interval and at places where ordered.

Gavirneni et al. (1999) compares the cases as non-shared information, partial information sharing, and full information sharing. In case of partial information sharing, the supplier knows the face-to-face distribution requests, orders are placed on a policy and specific parameters. In the full information sharing, the supplier retains the instant information requests from the retailer.

\subsection{Effect of Information Sharing in Supply Chain Management}

Over the last decade, supply chain management, co-operation, coordination and integration have become the focus of both academic and business studies. Collaboration in the supply chain has a great impact on the organizations' needs and cost reduction. Information sharing is one of the main strategies for eliminating the impact of the whip. There are extensive studies in the field of information sharing and coordination of Tayur et al. (1999) and Sahin and Robinson (2002) supply chain. In the Cachon and Fisherin (2000) studies, the value-added value of knowledge sharing and coordination is $35 \%$ of the total cost.

The rise in computer power enabled algorithm-based optimization approaches to address problems encountered in supply chain management (Aviv and Federgruen 1998, Gambling 2001). This has sparked the sharing of information between the firms and the integration of technology, thus creating new administrative approaches such as EDI, VMI and CPFR (Cachon, and Lariviere, 1999, Kleindorfer, and Wasselhove 2003).

Most of the work available on the types of shared information, and most researchers have demonstrated better performance in reducing the impact of intelligence sharing.

Simchi-Levi and colleagues (2004), who have been manufacturing a finishing capacity with a single retailer and a manufacturing chain, have stated that the purpose of their work is to analyze the value of IT sharing between these elements.

Most studies have concentrated on performance analysis on a 2-stage supply chain, with few studies studied multi-stage supply chains with various IT sharing scenarios.

Information sharing enhances supply chain performance. After the request, inventory and / or capacity information is shared, supply chain elements, inventory levels, inventory policies, capacity information and current demand information are aware. So the supply chain elements will receive more real-time and effective decisions. Service level, completion rate, and order return time will appear apparently. 
Collaboration is an important mechanism for increasing the supply chain. Collaboration reduces the effectiveness of the glass and increases productivity.

Sharing customer inventory information ensures that your own production and distribution schedules should be well-adapted to a good service level (Gavirneri v.d. 1999). This type of operation is called VMI (vendor-managed inventory) and is described in detail in Chapter 1. Common communication allows the supplier to respond to demand changes. If the retailer shares the customer information order with the manufacturer to fulfill the orders, the supplier will not have a benefit to the retailer, though the manufacturer will have a reduction in the inventory level (Li v.d.2001). However, if retailers bring the inventory change to the manufacturer, both the retailer and the manufacturer benefit from the reduction of inventory costs. In the level of IT sharing, the manufacturer benefits more than the retailer (Barut v.d. 2002).

Order information sharing provides good results in inventory sharing when orders are stable and sufficient (Cachon and Fisher 2000). Supplier can calculate the customer request using the sales rate information.

If the demand data sharing request is high, it is valuable for the retailer. The retailer will be more precise than the estimated manufacturer. Inter-estimate correlations will be low (Li 2001). On the other hand, demand information sharing will not be helpful if the demand change is high. The reason for this is that demand for information on the demand network will be low and the cost reduction will not be efficient (Gavirneni v.d. 1999, Li v.d. 2002). Predictive information sharing can be said to be particularly useful in the demand for more dynamic and customer-friendly industries.

Full demand information sharing is achieved through the demand supply network that contains all the steps downstream. Inventory control is usually the center. Full demand information sharing also reduces the effectiveness of the wheat and the safety stock. When the demand for consumer demand is low, full demand for IT dissertation falls below $75 \%$ of inventory levels ( $\mathrm{Li}$ et al 2001).

$\mathrm{Li}$ (2001) has 5 rules in the sharing of information. These guidelines are presented as follows.

- Share information that will only increase supply chain performance

- Simplify, synchronize and balance the demand, procurement planning processes.

- Use the different demand data resources combinations.

- Provide benefit from establishing common relations with customers

- $\quad$ Try to understand the basic needs of the suppliers for the demand information.

The information that companies share in traditional supply chain management is just the order information. But today, the information technologies provide the companies to share the demand and inventory data quickly and inexpensive. Information technologies have strong effects on the supply chain. Data collected from sales points can be easily shared in all stages of the supply chain through EDI. Informatics technologies that simplify IT sharing also reduces delay times and reduces process orders in time and cost in shipping frequency. Manufacturers and retailers are restructuring their supply chains by using information technologies such as EDI and POS systems. Information technology enables manufacturers and retailers to share information on demand and inventory levels.

Gavirneni (1999) and his colleagues shared their knowledge sharing to 3 levels. The level of informatics (NIS), the partial share sharing level (PIS) and the complete level of sharing (FIS). Suppliers at the level where the information is not shared view the inventory policy and demand information of retailers. Partial sharing levels see the inventory level and demand information of retailers. Gavirneni and colleagues have shown that the supply of PISs from NIS to PIS decreased by more than 50\%. From PIS to FIS, the suppliers' earnings were up to $35 \%$.

\subsection{Information Sharing and Coordination in Supply Chain Management}

Sahin et al. (2004) finds the aim of analyzing the interdependent system coordination and the sharing of knowledge sharing, finding benefits from information sharing or coordination, working on channel utilities, and analyzing external environmental effects to system cost performance. Experimental results have shown that a cost reduction of $47.58 \%$ between a conventional supply chain and a fully integrated system. While sharing of information sharing costs decreases, the main economic benefit comes from decision-making.

The development of information technology offers companies the opportunity to develop supply chain strategies and new research possibilities for international cooperation. Higher product values, demand for global markets, short product life cycles and customer service, supply chain complexity and cost increases.

In this research, the effects of system integration in the two perspectives of supply chain integration, IT sharing, and physical flow coordination and vendor-producer relationship were investigated.

In this study, a simulation study has been conducted to measure the impact of the sharing of information under a variety of scenarios in a multi-purpose supply chain. As a result of the experiments, the inventory and sequestration values made significant gains from the perspective of distributors and wholesalers.

The supply chain is a logistics network that includes all orders in order to produce and distribute a product or service (order processing, purchase, inventory control, production and distribution). All the chain; It is a network that produces products to consumers, retailers, retailers, distributors, suppliers, and suppliers. 
The share of both supply and demand information reduces inventory costs, based on stoic and order-based manufacturing. As a result of fully sharing of information, the supply chain performance is optimal, and each decision maker best suits your individual purpose (Sahin and Robinson 2002).

Information sharing substantially increases supply chain performance. Companies can redefine supply chain strategies by sharing their information with the sharing of information. Many researches on the supply chain have examined the positive effects of the sharing of information. Several studies have explored how the degree of knowledge sharing knowledge on the supply chain performance. In this study, the analysis of the supply chain performance measurement with different IT sharing scenarios has been made. Sharing is usually taken as the main item for cooperation in the supply chain. Higher estimates can be achieved if more direct and immediate information is available in a supply chain.

In the study of Chantrasa (2005), it has determined the benefits and risks of IT sharing according to the technical, management and partnership criteria. Technical benefits have been listed as a drop in inventory level, decline in processing time, production planning and scheduling, and reduction in supply chain costs. Administrative benefits have been reported to improve communication, as well as better decision-making in capacity building. The benefits of the partnership have been to facilitate supply chain coordination and to increase conflict resolution. Information sharing risks are based on the high cost and system maintenance costs in the adaptation of IT technologies according to technical criteria. According to administrative criteria, complex management has determined more time allocation and administrative control, flexibility and effectiveness to verify data.

According to the partnership criteria, more confidence has been shared with partners, losing partnership control and high cost building relationships with partners.

\subsection{The Result}

The significance of the sharing of information between the integrated supply chain elements and the benefits they bring from the detailed literary work presented above are explained. As a result of studies, it is seen that the studies conducted for acquisition of knowledge sharing in the supply chain networks have generally taken into consideration three or four stages of a few studies, which have often been acquired by supplying two-stage supply chains. The model presented has been developed to measure the sharing of knowledge in a five-stage integrated supply chain network and the information that needs to be shared based on previous studies has been set for each stage. Thanks to this model, the value of computing and IT acquisitions in the industry independent companies has been transformed into rules with the inductive learning method and has been made available to companies in the supply chain sharing capabilities. The information sharing company has been able to provide solutions to the problems they are experiencing in the supply chain, with the opportunity to have healthier, cooperative and cooperable supply chains.

According to the results of the literature survey, a model developed for measuring information in integrated supply chain networks was proposed and the function of the model was discussed.

Supply chain stemming and sharing of information sharing plays a critical role. This study has been developed for a model of sharing information between supply chain stages (knowledge sharing, supply chain dynamism, operating performance).

The study begins with the mathematical models of sharing information sharing to better understand relationships between supply chain members. The theorems obtained from mathematical models have been tested in more generalized supply chain arrangements. The development of the mathematical model will provide perspectives to the sharing of knowledge in the supply chain and help to develop experimental models.

\section{Definition of the Model}

IT sharing plays a crucial role in the supply chain management stages and its members. Different aspects of supply chain stages and information sharing have been studied in the supply chain management (Barut v.d. 2002, Fiala 2005, Mu Chen Chen et al., 2006, Mu Chen Chen et al., 2007). From this work, a model has been developed to measure the sharing of information between the proposed model and supply chain stages (knowledge sharing, supply chain dynamism, operating performance). The steps below are described in the steps described below. In addition, the schematic representation of the proposed model is presented in Figure 1.

In the first phase of the model, an integrated supply chain network is designed. Network components consist of suppliers, suppliers, distributors, retailer and customers. Then data was collected through the questionnaire method to obtain shared data between network elements. The questionnaire was created based on the work done by Barut (2000). Data from the five elements of the integrated supply chain are collected and the data is intended to be measured in the network thanks to these data. The questions are industry-independent and prepared in small, medium-sized and large enterprises. The questions are given in Appendix A. The results were obtained by applying the prepared survey to different businesses. Survey questions have been answered in the face, face, and virtual environment for people in the industry. Based on the evaluation of these results, consistent and appropriate polls 
were selected. According to these selected results, the appropriate data set has been created. The collected data was quantitatively and structural equations were established to convert qualitative.

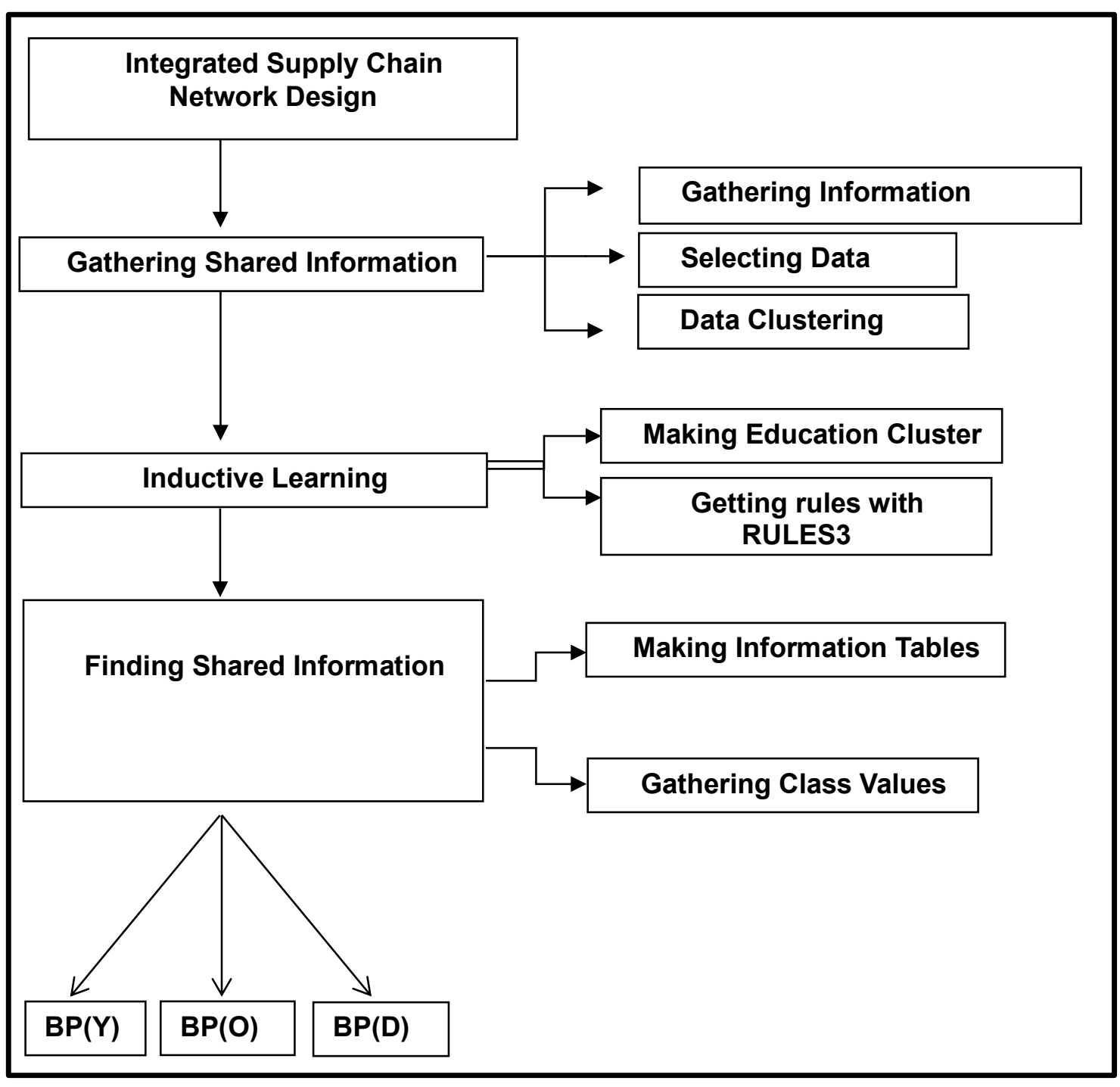

Figure 1. Schematic Representation of the Model Structure

The results were obtained by applying the prepared survey to different businesses. Survey questions have been answered in the face, face, and virtual environment for people in the industry. Based on the evaluation of these results, consistent and appropriate polls were selected. According to these selected results, the appropriate data set has been created. The collected data was quantitatively and structural equations were established to convert qualitative. The qualitative data are processed by the inductive learning method of artificial intelligence. The creation of training sets was created with the RULESX algorithm and the sharing of rules for sharing information. At the end of the rules, the value of shared information can be obtained as High (Y) medium (O) and low (D).

\subsection{Description of the Mathematical Model}

The structural equations of the mathematical model are integrated in questionnaire with the questionnaires. Structural equations are based on the study of Barut (2000). The method used by Barut's customer and supplier mapping has been developed and applied to the integrated supply chain network.

The integrated supply chain network elements and abbreviations are presented in the mathematical model as follows.

1. Supplier-T 2. Manufacturer-I 3. Distributor-D 4. Retailer-P 5. Customer-M

The general equation of the computing-sharing measurement model (BTZABP) in the integrated supply chain network is shown below.

BTZABPO = [IT scope, Information Density (BY)]

Information Scope (BK) 
The Information Scope (BK) represents the number of information levels that are considered when establishing production / logistics plans in a business. The mentioned level of information consists of suppliers, manufacturers, distributors, retailer and customer levels.

"0 or 1". Table 1 provides an example of IT scope of data coverage based on the number of information levels. For every value, at least one of the level of knowledge is enough to have 1.

Information Density (BY)

IT density; supplier, distributor and retailer, request information, inventory information, scheduling information, capacity information and order information. In the customer segment, the data intensity; product information, aftersales service information, credit information, campaign information and personal information. At least one of these levels means that the presence of data density varies. If none of these levels are available, the value is taken as 0 . Table 2 shows the example of the data density values.

$$
\begin{aligned}
& B Y=V_{1} * B Y^{T}+V_{2} * B Y^{I}+V_{3} * B Y^{D}+V_{4} * B Y^{P}+V_{5} * B Y^{M} \\
& V_{1}, V_{2}, V_{3}, V_{4}, V_{5} \text { Is the weight of "0 or 1". }
\end{aligned}
$$

\subsection{Inductive Learning Method}

In this step of the proposed model, quantitative data collected by the survey method is intended to be transformed into qualitative data using the structural equation. The data obtained by the survey method will be evaluated using the inductive learning method. The inductive learning method is one of the artificial intelligence methods and has been decided to be used in this study as a result of the literature research. The ease of application of the inductive learning method and the achievement of the correct decisions demonstrates the necessity of using this method in the model. RULESX algorithm, one of the inductive learning methods, will be selected. As a result of the use of this method, it is aimed to determine the sharing of information on the integrated supply chain network and determine the limits. After all, the rules of the model will be obtained.

\subsection{Use of Inductive Learning in the Measurement of Information Sharing}

The inductive learning method is a set of decision trees or rules. The rules are easily obtained from the decision tree. Each branch of the decision tree is considered as a rule. The result of the inductive learning method is to obtain the most common rules possible. The RULESX algorithm is controlled by one by one, by controlling the rules that are most common, and the most common algorithm is the algorithm [Aksoy 2005]. In the model, the RULESX algorithm was preferred because of its advantages and application conveniences.

RULES-3 algorithm: The inductive learning method examines two parts as the decision-maker producing and directing rules. Decision-based techniques work with the principle of separation of subsystems. So, it divides the set of samples into sub-clusters and then creates a decision tree that moves from these subsets. The output of these techniques is a decision tree. CLS and ID3 are important sample algorithms for this class (Gill and Boctor 1997; Aksoy 2005; Aksoy 2005b;). The direct rule algorithms are algorithms that direct the rule without creating a decision tree as it is understood. Examples of these are AQ and RULES. Algorithms in both classes are important in applied artificial intelligence. The RULES algorithm has 4 different versions; RULES-1, RULES-2, RULES-3, and RULESX. RULES's expansion is RULE Extraction System (Over 2006).

The RULES-3 algorithm selects the most common one by checking the rules that can be obtained. In short, the rules that can classify the sample are examined after each of them has classified the few samples in the same class, in the same set of samples. The rule that can classify the most common example is generally accepted and selected.

The basic steps of RULES-3 are as follows (Aksoy 2005);

Step 1. Define the ranges for the numeric value that contains the value, Step 2. Identify the minimum number of conditions (descriptions) for the rules to be removed, Step 3. Identify the number of rules that can be extracted for each sample Step 4. Select an unclassified example, Step 5. Nc $=$ Stop -1 , Step 6. If $\mathrm{Nc}<\mathrm{Na}$ is $\mathrm{Nc}=\mathrm{Nc}+1 \mathrm{Step}$ 7. Create a set of values with the characteristic values used in the example shown in the example. Step 8. Find Nc's combinations of elements in the array of arrays. Step 9. If at least one of these combinations is passed in only one class in all the samples in the control panel, make it a rule, otherwise go to Step 6. Step 10. Choose the most general one from the selected rules. Step 11. Extract samples that can be classified by the selected rule.

Step 12. If all the samples are classified, then go to step 4. Where $\mathrm{Nc}$ is the number and the $\mathrm{Na}=$ characteristic number $[1,2]$. If the specifics containing the numeric values are created in the setup samples, the ranges are defined in Step1. However, rules can be generated in the set of samples that contain numerical value characteristics. The steps of this process are accomplished as follows;

First, the smallest and largest values found in the samples given for numerical values are detected. Then, by the expert opinion or from the literature, ranges between these values are determined and the set of samples are created according to these labels by giving a verbal tag to each range. A rule may include at least one, maximum, and the total characteristic number. In Step2, the minimum required number is required to have a rule. In step 3, the number of rules that you want to remove for each sample is determined. In Example 4, an example randomly selected from 
the sample set is randomly selected. The number of conditions for the sample selected in step 5 and step 6 is regulated. The value used in Example 7 is assigned to a series. Step8 has the combinations of this directory. At step 9, each test will be tested for each rule. If this combination is only a class in a combination set of samples, this example is set as a rule. If no rule is found, the number of conditions is increased. This process is repeated continuously until at least one rule is found. In Step 10, if there is more than one rule in the preceding step, one of the samples is selected from the set of samples from the set of samples. Steps that can be classified under selected rules are extracted from step 11. In step 12, the algorithm stops if the rule has not been explicitly set out for that rule, if it is left, the steps are returned to Step 4 and the process repeats. Finally, all the rules obtained are brought together and the information base is created. This information base is the generalized form of the sampling set.

As seen from the steps of the algorithm, it is a process that requires repeating rules from the set of samples. According to the rules set out in this program, the computer program can decide which rules to take on new rules when given similar examples, seeing you as a specialist. The RULESX algorithm is a new version of the RULES3 algorithm, which is a more functional version and is used in the study.

Table 1 shows the linguistic expressions of the scope of value ranges of information. The minimum and maximum values, which are the values of the resultant solutions, are calculated as $(0,1)$.

\begin{tabular}{|l|l|l|}
\hline Number of Example & Value Ranges & Linguistic expressions \\
\hline 1 & $0<\mathrm{D} \leq 0,5$ & Lowest(ED) \\
\hline 2 & $0.5<\mathrm{D} \leq 1,5$ & Low(D) \\
\hline 3 & $1.5<\mathrm{D} \leq 2,5$ & Mid Low(OD) \\
\hline 4 & $2,5<\mathrm{D} \leq 3,5$ & Mid (O) \\
\hline 5 & $3.5<\mathrm{D} \leq 4$ & Mid High (OY) \\
\hline 6 & $4<\mathrm{D} \leq 4,5$ & High (Y) \\
\hline 7 & $4.5<\mathrm{D} \leq 5$ & Highesy (EY) \\
\hline
\end{tabular}

Table 1. BK Linguistic Representation of Value Ranges

Value ranges for IT density (BY) are determined and the values falling in these ranges are shown in Table 2. The value of the data density value was calculated as the lowest and highest values (0.99). According to this calculation, the Table 2 was created. It is intended to prevent loss of data with specified range ranges. Information intensity and the scope of the information are provided with similar expressions.

\begin{tabular}{|l|l|l|}
\hline Range Numbers & Value Ranges & Value \\
\hline 1 & $0 \leq \mathrm{D} \leq 10$ & 0.5 \\
\hline 2 & $10<\mathrm{D} \leq 25$ & 1 \\
\hline 3 & $25<\mathrm{D} \leq 35$ & 1.5 \\
\hline 4 & $35<\mathrm{D} \leq 40$ & 2 \\
\hline 5 & $40<\mathrm{D} \leq 45$ & 2.5 \\
\hline 6 & $45<\mathrm{D} \leq 55$ & 3 \\
\hline 7 & $55<\mathrm{D} \leq 60$ & 3.5 \\
\hline 8 & $60<\mathrm{D} \leq 75$ & 4 \\
\hline 9 & $75<\mathrm{D} \leq 85$ & 4.5 \\
\hline 10 & $85<\mathrm{D} \leq 99,8$ & 5 \\
\hline
\end{tabular}

Table 2. BY Value Ranges and Values

Linguistic expressions of value range of the information density are described in Table 3. Considering the value ranges in Table 2., these values are indicated by linguistic expressions. 


\begin{tabular}{|l|l|l|l|}
\hline Number of Example & Value Ranges & $\begin{array}{l}\text { Linguistic expressions } \\
\text { Values }\end{array}$ & Linguistic expressions \\
\hline 1 & $0 \leq \mathrm{D} \leq 10$ & 0.5 & Lowest (ED) \\
\hline 2 & $10<\mathrm{D} \leq 25$ & 1 & Low(D) \\
\hline 3 & $25<\mathrm{D} \leq 35$ & 1.5 & Low(D) \\
\hline 4 & $35<\mathrm{D} \leq 40$ & 2 & Mid Low(OD) \\
\hline 5 & $40<\mathrm{D} \leq 45$ & 2.5 & Mid Low (OD) \\
\hline 6 & $45<\mathrm{D} \leq 55$ & 3 & Mid (O) \\
\hline 7 & $55<\mathrm{D} \leq 60$ & 3.5 & Mid High (OY) \\
\hline 8 & $60<\mathrm{D} \leq 75$ & 4 & Mid High (OY) \\
\hline 9 & $75<\mathrm{D} \leq 85$ & 4.5 & High (Y) \\
\hline 10 & $85<\mathrm{D} \leq 99,8$ & 5 & Highest (EY) \\
\hline
\end{tabular}

Table 3. BY Linguistic Representation of Value Ranges

\begin{tabular}{|l|l|}
\hline $\begin{array}{c}\text { Information Sharing } \\
\text { Linguistic expressions }\end{array}$ & $\begin{array}{c}\text { Information Sharing } \\
\text { General Linguistic expressions }\end{array}$ \\
\hline (D, ED) & D \\
\hline (EY, EY) & Y \\
\hline (OY, OD) & OD \\
\hline (OD, D) & D \\
\hline (OD, D) & D \\
\hline (D, D) & D \\
\hline (OD, D) & D \\
\hline (OD, OD) & OD \\
\hline (OY, O) & O \\
\hline (OY, OD) & O \\
\hline (OY, Y) & OY \\
\hline (O,OY) & OY \\
\hline (OD, D) & D \\
\hline (OD, D) & D \\
\hline (O,OD) & D \\
\hline (O,O) & O \\
\hline (OY, Y) & OY \\
\hline (O,OY) & OY \\
\hline
\end{tabular}

Table 4. Showing BP with General Linguistic Expressions

As a result, it was seen that the low-level (D) medium (O) and high (Y) stages of the medium (O) were mentioned in the light of the information sharing, the scope of information and the data density. As a result of the rules set out by the inductive learning method, the general linguistic expression of information sharing has emerged as medium (O).

\subsection{Analysis Findings}

According to the model offered by the firms' supply chains, shared information on each stage of the chain, the flow of the share and the recommendations presented below are presented below.

Demand information: Demand information is one of the important information that should be shared in the supply chain. It is important to share this information with the fact that members of the individual individually demanding a supply chain cause the camcorder effect. There are 17 companies in the survey which have a share of demand information with the supplier level. Distributor phase and the sharing of the demand information are available in 6 companies and receive the lowest value. Specifically, the sharing of information with programs such as Collaboration, Predictive and Renewal (CPFR) may be healthier than supply chain members.

Inventory Information: Organizations in the supply chain can encounter various problems if they manage their inventory independently without information sharing. The control system known as "stock-based inventory control" is used to perform shared inventory level information. In this way, moving companies can decide when and what they will produce. It has been shown that the share of inventory information is reached with the manufacturer's level of 10 firms.

Capacity Information: Sharing capacity information in the supply chain is a matter which is important for determining chain integration and suppliers. Sharing the resource capacity information will reduce the potential effect of the camel. In the supply chain, firms can coordinate and protect potential shortcomings when they share 
capacity information. Within the firms in which the survey is implemented, it is seen that the share of capacity information in the supplier and producer phase is greater.

Production Scheduling Information: Sharing Scheduling Information enables organizations to develop their own production charts among sharing members. The information about the supplier's production will benefit the vendor's planning horizons and the realistic scheduling of your own scheduling schedule. This allows the seller to identify more accurate dates with their customers. When we examine the companies in which the survey is applied, the share of the scheduling information at the supplier level is higher than the 13 companies and the other stages.

Order Information: Order information is information that must be shared every step of the supply chain. It is very important to share the fact that the effect of the wheat in the flow of information from the customer to the supplier is unprecedented. According to the results of the survey, it is observed that the share of order information in 13 suppliers in the supplier, distributor and distributor stages.

Personal Information: Sharing the personal information of customers on the supply chain network is one of the important criteria when planning sales. It is important to keep these data in the database, as well as the convenience of being prompted, to deliver products to customers. According to the results of the survey, the 13 firms found that the share of personal information is reflected in their sales plans at the customer level

Post-Sales Service Information: Share after sales service is one of the important information that should be available in sales plans. Important criteria can be made to examine the products sold, sharing customer satisfaction by sharing this information. Survey results show that post-sale service information is shared by 7 companies.

Credit Information: Credit information is one of the important information used in the planning of product sales and is important to share. By sharing this information, the customer portfolio can be removed and analyzed whether the customer is eligible for credit sales. It is seen that 7 companies participating in the survey are planning to use this information when planning.

Campaign Information: Campaigns that previously participated in the campaign or the planned campaign information planned to be made in the future is one of the information that should be considered when planning sales. Only 4 companies participating in the survey are using this information in their sales plans.

Product information: Share of product information is important for the customer to meet and meet demands. Within the scope of the survey, 17 firms share product information. Share of product information is a fact that customers are significantly influencing their purchasing behavior positively.

The result: It is seen that there is information sharing within the firms, but that the flow of information is not in a certain order, and that this disorder is low in terms of knowledge sharing, and that these levels can be upgraded within the proposed model. As a result of the survey, it is found that the sharing of information sharing with the retail segment, especially in the retail chain, is very weak, and in the producer and supplier phase it is better to know that sharing information is better than other stages. With this proposed model, the sharing of data sharing is realized and it is believed that supply chain software can be brought to a new dimension with this model.

\section{References}

- Aksoy, M. S., "Pruning Decision Trees Using Rules3 Inductive Learning Algorithm”, Mathematical and Computational Applications, Vol. 10, No. 1, pp. 113-120, 2005

- Aksoy, M. S., "Dynamic System Modeling Using Rules3 Induction Algorithm”, Mathematical and Computational Applications, Vol. 10, No. 1, pp. 121-132, 2005

- $\quad$ Aviv, Y., A. Federgruen. (1998). "The Operational Benefits of Information Sharing and Vendor Managed Inventory (VMI) Programs”. Working Paper http://www.olin.wustl.edu/faculty/aviv/

- Barut M. Faisst. W. Kanet J. J. "Measuring supply chain coupling: an information system perspective" European Journal of Purchasing \& Supply Management, Volume 8, Issue 3, September 2002, Pages 161171

- Cachon, G.P. and M. Fisher (2000)." Supply Chain Inventory Management and the Value of Shared Information.” Management Science, 46,No. 8 pp:1032-1048.

- Chantrasa R.(2005) "Decision Making Approaches for Information Sharing in a Supply Chain" Ph.D. Thesis The Graduate School of Clemson University Umi number: 3183171

- Fiala P., (2005) "Information sharing in supply chains" Omega, Volume 33, Issue 5, October 2005, Pages 419-423

- Gavirneni, S., Kapuscinski, R., Tayur, S.(1999): "Value of information in capacitated supply chains". Manage. Sci. 45(1), 16-24

- Gunasekaran A., Ngai E.W.T., (2004)“Information Systems in Supply Chain Integration and Management” European Journal of Operational Research 159 269-295 
- Guo Z., Fang F.. Whinston A. B. (2006) "Supply chain information sharing in a macro prediction market" Decision Support Systems, Volume 42, Issue 3, Pages 1944-1958

- Kalakota, R., Robinson, M. (2001) “E-business 2.0.” Addison-Wesley, 2001.- 520 p.

- Kleindorfer, P. R., and van Wasselhove, L. (2003) "Managing Risk in Global Supply Chains” Working paper, Wharton School, University of Pennsylvania,.

- Lee H.L., and S. Whang. (1998) “Information Sharing in a Supply Chain.” Research Paper No.1549, Graduate School of Business, Stanford University.

- Li, L. (2002) "Information Sharing in a supply Chain with horizontal Competition", Management science 48 no.9, , pp.1196-1212.

- $\quad$ Li, J., M.J. Shaw, R.T. Sikora, G.W. Tan, and R. Yang, (2001). "The Effects of Information Sharing Strategies on Supply Chain Performance", College of Commerce and Business Administration, University of Illinois at Urbana-Champaign, URL: http://citebm.cba.uiuc.edu/B2Bresearch/ieee_em.pdf

- Mu-Chen Chen, Ming-Miin Yu, Shih-Chan Ting (2006) "Evaluating The Cross-Efficiency Of Information Sharing In Supply Chain Management "Proceedings of International Congress on Logistics and SCM Systems

- Mu-Chen Chen, Yang T. Yen C. T. (2007) "Investigating the value of information sharing in multi-echelon supply chains” RESEARCH NOTE Qual Quant DOI 10.1007/ s11135-007-9086-2 2007

- Sahin, F. And E.P. Robinson. (2002) “Flow Coordination and Information Sharing in Supply Chains: Review, Implications and Directions for Future Research.” Decision Sciences, 33,No.4, pp:505-536

- Simatupang, T. M. and Sridharan, R.(2001), "A Characterization of Information Sharing in Supply Chains" Proceedings of the 36th Annual ORSNZ Conference, pp.16-25.

- $\quad$ Simatupang, T.M., Sridharan, R. (2002), "The collaborative supply chain", The International Journal of Logistics Management, Vol. 13 No.1, pp.15-30.

- Simchi-Levi, D. and Y. Zhao (2004). "The Value of Information Sharing in a Two-Stage Supply Chain with Production Capacity Constraints: The Infinite Horizon Case". Probability in Engineering and Information Sciences 18: 247-274.

- Stefansson, G. (2002), "Business-to-business data sharing: A source for integration of supply chains", International Journal of Production Economics, Vol. 75, No. 1-2, pp. 135-146.

- Stevens, G. C. (1989) “'Integrating the supply chain". International Journal Of Physical Distribution And Materials Management, Cilt: 19, No: 8, Sf: 3-8,.

- Themistocleous M. , Irani Z., Love E. D (2004) "Evaluating the integration of supply chain information systems: A case study” European Journal of Operational Research, Volume 159, Issue 2, Pages 393-405 